\title{
COUPLING OF RADIAL AND NON-RADIAL OSCILLATIONS OF NEUTRON STARS.
}

\author{
Andrea Passamonti, ${ }^{1}$ Marco Bruni, ${ }^{1}$ Leonardo Gualtieri, ${ }^{2}$ and Carlos F. Sopuerta ${ }^{1,3}$ \\ ${ }^{1}$ ICG, University of Portsmouth, Portsmouth PO1 2EG, Britain \\ ${ }^{2}$ Dipartimento di Fisica "G. Marconi”, Universitg di Roma "La Sapienza" and Sezione INFN \\ ROMA 1, piazzale Aldo Moro 2, I-00185 Roma, Italy \\ ${ }^{3}$ IGPG and CGWP, The Pennsylvania State University, University Park, PA 16802, USA
}

\begin{abstract}
This is a progress report on our study of the coupling of first-order radial and non-radial relativistic perturbations of a static spherical star. Our goal is to investigate the effects of this coupling on the gravitational wave signal of neutron stars. In particular, we are looking for the existence of resonances and parametric amplifications, changes in the damping time of non-radial oscillations, etc. To that end, we have developed a formalism that introduces gauge invariant quantities to describe the coupling. Their equations have the same structure as the equations for first-order non-radial perturbations plus some source terms, which makes them very appealing for time domain studies.
\end{abstract}

\section{Introduction}

Neutron stars are important gravitational wave sources both as isolated objects or in binary systems. The analysis of their gravitational radiation could open up a direct window on their interior, possibly revealing details on the equation of state of nuclear matter, the dynamics of the crust-mantle interaction and the inner superfluid/superconducting core. An accurate modelling of sources is crucial to the final end of providing templates of the wave form and spectrum of the signal for the analysis of data that comes from the new generation of gravitational wave detectors.

Linear perturbations and instabilities of neutron stars have been studied for a long time [1] but relatively little is known of non-linear dynamical effects (see [2] for references) and therefore second order studies may help to understand known problems and even reveal a new phenomenology.

Here we outline our work in [2], where we introduced a framework to study the coupling of radial and non-radial first-order relativistic perturbations of static spherical stars. The goal is to study effects of this coupling (possible res- 
onances, parametric amplification, etc.) that could make a significant impact in the gravitational wave signal of neutron stars.

\section{Perturbative Framework}

Using 2-parameter relativistic perturbation theory $[3,4]$ we parametrize separately the radial and non-radial perturbations. This allows us to split second order perturbations into three differentiated types: second-order radial and non-radial and the coupling between them. We are interested in the third type.

The basic ingredients of our perturbative framework are: (i) The background model for the star. We use a Tolman-Oppenheimer-Volkov model, that is, an equilibrium perfect-fluid spherically symmetric configuration. (ii) First-order radial perturbations (see [5] and references therein). These are perturbations preserving the spherical symmetry of the background model. They can be described by just three functions: two matter variables (in our case we choose the enthalphy and the fluid velocity radial component perturbations) and a metric variable. They obey a system of evolution equations containing only first-order time derivatives and subject to a constraint, the Hamiltonian constraint, which can be used to monitor the accuracy of a time-domain integration. (iii) Firstorder non-radial perturbations. Due to the symmetry of the background, these perturbations can be expanded in terms of (tensor) harmonics, so that the angular dependence is explicitly separated. Depending on how they behave under parity transformations they are called polar (even) or axial (odd) perturbations. Every harmonic (the monopole part would correspond to the radial perturbations, so it is not consider here) can be described in terms of gauge-invariant variables by using the formalism developed in [6-8]. These perturbations have been extensively studied in the literature due their interest in relation with the gravitational radiation produced by star oscillations. Most of the works in the past were done in the frequency domain [9-11]. However, recent works have studied them by using a time-domain approach [12-14]. (iv) Coupling of radial and non-radial perturbations. As we have mentioned above, these perturbations are a part of the second-order perturbations of our background. The part that is generated by the coupling of the radial and non-radial firstorder perturbations. This is the sector of the second-order perturbations that we have to study in order to look for the physical phenomena we described in the abstract and introduction, and it is the subject of [2]. The structure of these perturbations is very particular. By pure inspection of Einstein's equations one can see that they are generated by source terms that can be expressed as a sum of products of radial and non-radial first order perturbations. This means that they can be also expanded in (tensor) harmonics, which makes the analysis much simpler than the analysis of the whole set of second-order perturbations. Moreover, in [2] we were able to show that one can also have a gauge-invariant 
description just by extending in an appropriate way (in particular, by fixing the gauge for the radial perturbations) the formalism for non-radial perturbations introduced in [6-8].

Going into more detail about the structure of the equations for the coupling perturbations, we have seen in [2] that these perturbations, once decomposed in harmonics, obey the same equations as non-radial perturbations do, with the only difference that for the coupling terms we have source terms which, as we mentioned before, can be written as the sum of products of radial and nonradial perturbations. This structure has very important consequences for practical purposes, in particular for the numerical integration of the equations. Indeed, given a numerical code capable of evolving the non-radial perturbations we can construct a code evolving the coupling perturbations just by adding the sources. Having this interesting property in mind, an appropriate way of formulating (choice of variables and equations) the equations for non-radial perturbations would be the one choosen in [14], where the Hamiltonian constraint (an elliptic-type equation) is used to solve for one of the perturbative variables instead of using an evolution equation. Apart from the obvious interest that this procedure has (we make sure that constraints are preserved during the evolution), it has an extra interest thinking on solving also for the coupling perturbations: If we do not solve for the Hamiltonian constraint the errors produced by its violation would increase since we would accumulate the ones coming from the integration of the non-radial perturbations with the ones after solving for the coupling. Therefore, solving for the Hamiltonian constraint can improve substantially the accuracy of the calculations. As it has been shown for the case of non-radial perturbations it can estimate damping times and mode frequencies with an accuracy comparable to frequency domain calculations [14]. Hence, the structure of the system of equations governing the stellar interior is given by a gravitational wave equation for the non conformal-flat metric perturbation $S$, a sound wave equation for a fluid perturbation $H$ (coincident with the enthalpy perturbation in some particular gauges), and finally the Hamiltonian constraint mentioned above, used to update the value of the conformal-flat metric perturbation $k$ at every time-step,

$$
\begin{aligned}
-S_{, t t} & +e^{2(\Phi-\Lambda)} S_{, r r}+\ldots \ldots=e^{2 \Phi} \mathcal{S}_{S} . \\
-H_{, t t} & +\bar{c}_{s}^{2} e^{2(\Phi-\Lambda)} H_{, r r}+\ldots \ldots=e^{2 \Phi} \mathcal{S}_{H}, \\
k_{, r r} & +\frac{2}{r \bar{c}_{s}^{2}}\left(\Lambda_{, r}+\Phi_{, r}\right) H+\ldots \ldots=\mathcal{S}_{\text {Hamil }},
\end{aligned}
$$

where $\mathcal{S}_{S}, \mathcal{S}_{H}, \mathcal{S}_{\text {Hamil }}$ are the source terms containing the product of first order radial/nonradial perturbations [2].

To sum up, our framework to study the coupling of radial and non-radial oscillations leads to a hierarchy of equations (from the background to the coupling terms). In order to solve them in the time domain we have to pay attention 
to a number of other important issues. Of particular relevance are the boundary conditions: We need to impose the regularity of the perturbations at the origin, the vanishing of the Lagrangian perturbation of the pressure at the star surface, and the continuity of metric perturbations at the surface (junction conditions), connecting with the exterior, which can be described by the corresponding Zerilli equation, which will propagate the gravitational wave signal. Then, we can use the well-known black-hole perturbation machinery to compute the energy and angular momentum that has been radiated away. A discussion of all these issues can also be found in our first work [2].

\section{2. $\quad$ Future work}

We are presently working in the construction of numerical codes for the time domain integration of the equations derived in [2]. In this work we focused on polar perturbations, which are the most relevant for stars, but we are also exploring the axial case. On the other hand, this work can also be considered as a step towards a more comprehensive study of second order perturbations of compact stars and mode coupling.

\section{Acknowledgments}

This work has been partially supported by the EU (Research Training Network contract HPRN-CT-2000-00137). CFS was supported by EPSRC and presently by NSF grants PHY-9800973 and PHY-0114375.

\section{References}

[1] N. Andersson, Class. Quant. Grav. 20, R105 (2003).

[2] A. Passamonti, M. Bruni, L. Gualtieri, and C.F. Sopuerta, (2004), gr-qc/0407108.

[3] M. Bruni, L. Gualtieri, and C.F. Sopuerta, Class. Quant. Grav. 20, 535 (2003).

[4] C.F. Sopuerta, M. Bruni, and L. Gualtieri, Phys. Rev. D 70, 064002 (2004).

[5] K.D. Kokkotas and J. Ruoff (2002), gr-qc/0212105.

[6] U.H. Gerlach and U.K. Sengupta, Phys. Rev. D 19, 2268, (1979).

[7] C. Gundlach and J.M. Martin-Garcia, Phys. Rev. D 61, 084024 (2000).

[8] J.M. Martin-Garcia and C. Gundlach, Phys. Rev. D 64, 024012 (2001).

[9] K.S. Thorne and A. Campolattaro, Astrophys.J. 149, 591 (1967).

[10] S. Detweiler and L. Lindblom, Astrophys.J. 292, 12 (1985).

[11] S. Chandrasekhar and V. Ferrari, Proc. Roy. Soc. (London) A 432, 247 (1991).

[12] G. Allen, N. Andersson, K.D. Kokkotas, and B.F. Schutz, Phys. Rev.D 58, 124012 (1998).

[13] J. Ruoff, Phys. Rev.D 63, 064018 (2001).

[14] A. Nagar, G. Diaz, J.A. Pons, and J.A. Font, Phys. Rev. D 69, 124028 (2004); A. Nagar and G. Diaz, (2004), gr-qc/0408041. 